\title{
Efficient evaluation of partition functions of frustrated and inhomogeneous spin systems
}

\author{
V. Murg ${ }^{1}$, F. Verstraete ${ }^{1,2}$, J. I. Cirac ${ }^{1}$ \\ ${ }^{1}$ Max-Planck-Institut für Quantenoptik, Hans-Kopfermann-Str. 1, Garching, D-85748, Germany \\ ${ }^{2}$ Institute for Quantum Information, Caltech, Pasadena, US
}

(Dated: September 8, 2018)

\begin{abstract}
We present a numerical method to evaluate partition functions and associated correlation functions of inhomogeneous 2-D classical spin systems and 1-D quantum spin systems. The method is scalable and has a controlled error. We illustrate the algorithm by calculating the finite-temperature properties of bosonic particles in 1-D optical lattices, as realized in current experiments.
\end{abstract}

PACS numbers: 03.67.-a, 03.75.Lm, 02.70.-c, 75.40.Mg

The study of thermodynamic properties of 2-D classical spin systems has a very long and fruitful history. On the one hand, it has provided valuable insights in the theory of magnetism and phase transitions. On the other, it has allowed us to describe 1-D quantum systems at finite temperature by virtue of the Suzuki-Trotter decomposition.

As very few spin models are exactly solvable, many different approximate methods have been proposed to calculate the associated partition functions. Monte Carlo seems to be the method of choice for non-frustrated systems, but fails in the description of frustrated and fermionic systems due to the notorious sign problem [1]. No such sign problem occurs in the method developed by Nishino 2], where the largest eigenvalue of the transfer matrix of the classical spin model can be approximated by using a variation of the density matrix renormalization group (DMRG) approach 3, 4]. By making use of the Suzuki-Trotter decomposition, this method has also been used to calculate the free energy of translational invariant 1-D quantum systems [5, 6, 7]. The main restriction of this method is that it cannot be applied in situations in which the number of particles is finite and/or the system is not homogeneous. The method may also become ill conditioned when the transfer matrix is not hermitian. Finally, in the case of 1-D quantum systems, a recent development [8, 9] allows us to overcome these problems by extending the concept of matrix product states 10, 11. to mixed states, e.g. by using the idea of purification of states 8]. This method is, however, specially designed for $1-\mathrm{D}$ quantum systems, and cannot be extended to classical 2-D models.

Here we take a completely different approach which allows us to overcome the drawbacks of the above mentioned methods in both 2-D classical and 1-D quantum systems. We achieve this by evaluating the associated partition and correlation functions directly. The main advantages of this method are that the approximations made are very well controlled, that it applies to frustrated, inhomogeneous and finite classical and quantum systems, and that it can be generalized to higher dimensions. We will illustrate its performance with the system of strongly interacting bosonic atoms in optical lattices, a problem which has attracted a lot of interest in the atomic physics community in the last few years due to the recent experimental achievements [12, 13, 14]. In this system, 20-100 atoms are trapped by the combination of a periodic and a harmonic (i.e. inhomogeneous) potential created by lasers in $1-\mathrm{D}$ and at a finite temperature. In the so-called Tonks-Girardeau limit 15], the problem can be exactly solved via fermionization 13], and thus this provides us with a reliable benchmark for our method. Outside this limit, we are able to reproduce certain features experimentally observed [12].

Our method relies in reexpressing the partition and correlation functions as a contraction of a collection of 4 -index tensors, which are disposed according to a $2-\mathrm{D}$ configuration. We will perform this task for both 2-D classical and 1-D quantum systems. We will then show how the methods introduced in [16] can be used to approximate these contractions in a controlled way, and thus lead to a scalable algorithm for the evaluation of the quantities of interest.

1.- 2-D classical systems: Let us consider first the partition function of an inhomogeneous classical 2-D n-level spin system on a $L_{1} \times L_{2}$ lattice. For simplicity we will concentrate on a square and nearest-neighbor interactions, although our method can be easily extended to other short-range situations. We have

$$
Z=\sum_{x^{11}, \ldots, x^{L_{1} L_{2}}} \exp \left[-\beta H\left(x^{11}, \ldots, x^{L_{1} L_{2}}\right)\right]
$$

where

$$
H\left(x^{11}, \ldots\right)=\sum_{i j}\left[H_{\downarrow}^{i j}\left(x^{i j}, x^{i+1, j}\right)+H_{\rightarrow}^{i j}\left(x^{i j}, x^{i, j+1}\right)\right]
$$

is the Hamiltonian, $x^{i j}=1, \ldots, n$ and $\beta$ is the inverse temperature. The singular value decomposition allows us to write

$$
\exp \left[-\beta H_{q}^{i j}(x, y)\right]=\sum_{\alpha=1}^{n} f_{q \alpha}^{i j}(x) g_{q \alpha}^{i j}(y)
$$


with $q \in\{\downarrow, \rightarrow\}$. Defining the tensors

$$
X_{l r u d}^{i j}=\sum_{x=1}^{n} f_{\downarrow d}^{i j}(x) g_{\downarrow u}^{i-1, j}(x) f_{\rightarrow r}^{i j}(x) g_{\rightarrow l}^{i, j-1}(x),
$$

the partition function can now be calculated by contracting all 4-index tensors $X^{i j}$ arranged on a square lattice in such a way that, e.g., the indices $l, r, u, d$ of $X^{i j}$ are contracted with the indices $r, l, d, u$ of the respective tensors $X^{i, j-1}, X^{i, j+1}, X^{i-1, j}, X^{i+1, j}$. In order to determine the expectation value of a general operator of the form $O\left(\left\{x^{i j}\right\}\right)=Z \prod_{i j} O^{i j}\left(x^{i j}\right)$, one just has to replace each tensor $X^{i j}$ by

$$
X_{l r u d}^{i j}\left(O^{i j}\right)=\sum_{x=1}^{n} O^{i j}(x) f_{\downarrow d}^{i j}(x) g_{\downarrow u}^{i-1, j}(x) f_{\rightarrow r}^{i j}(x) g_{\rightarrow l}^{i, j-1} .
$$

2.- 1-D quantum systems: We consider the partition function of an inhomogeneous 1-D quantum system composed of $L n$-level systems,

$$
Z=\operatorname{Tr} \exp (-\beta H) .
$$

It is always possible to write the Hamiltonian $H$ as a sum $H=\sum_{k} H_{k}$ with each part consisting of a sum of commuting terms. Let us, for simplicity, assume that $H=H_{1}+H_{2}$ and that only local and 2-body nearest neighbor interactions occur, i.e. $H_{k}=\sum_{i} O_{k}^{i, i+1}$ and $\left[O_{k}^{i, i+1}, O_{k}^{j, j+1}\right]=0$, with $i, j=1, \ldots, L$. The more general case can be treated in a similar way. Let us now consider a decomposition

$$
\exp \left(-\frac{\beta}{M} O_{k}^{i, i+1}\right)=\sum_{\alpha=1}^{\kappa} \hat{S}_{k \alpha}^{i} \otimes \hat{T}_{k \alpha}^{i+1} .
$$

The singular value decomposition guarantees the existence of such an expression with $\kappa \leq n^{2}$. As we will see later, a smart choice of $H=\sum_{k} H_{k}$ can typically decrease $\kappa$ drastically. Making use of the Suzuki-Trotter formula 25]

$$
Z=\operatorname{Tr}\left(\prod_{k} \exp \left(-\frac{\beta}{M} H_{k}\right)\right)^{M}+\mathrm{O}\left[\frac{1}{M}\right]
$$

it can be readily seen that the partition function can again be calculated by contracting a collection of 4-index tensors $X^{i j}$ defined as

$$
X_{\left(l l^{\prime}\right)\left(r r^{\prime}\right) u d}^{i j} \equiv\left[\hat{T}_{1 l}^{j} \hat{S}_{1 r}^{j} \hat{T}_{2 l^{\prime}}^{j} \hat{S}_{2 r^{\prime}}^{j}\right]_{[u d]},
$$

where the indices $\left(l, l^{\prime}\right)$ and $\left(r, r^{\prime}\right)$ are combined to yield a single index that may assume values ranging from 1 to $\kappa^{2}$. Note that now the tensors $X^{i j}$ and $X^{i^{\prime} j}$ coincide, and that the indices $u$ of the first and $d$ of the last row have to be contracted with each other as well, which corresponds to a classical spin system with periodic boundary conditions in the vertical direction. A general expectation value of an operator of the form $O=Z O^{1} \otimes \cdots \otimes O^{N}$ can also be reexpressed as a contraction of tensors with the same structure: it is merely required to replace each tensor $X^{1 j}$ in the first row by

$$
X_{\left(l l^{\prime}\right)\left(r r^{\prime}\right) u d}^{1 j}\left(O^{j}\right)=\left[O^{j} \hat{T}_{1 l}^{j} \hat{S}_{1 r}^{j} \hat{T}_{2 l^{\prime}}^{j} \hat{S}_{2 r^{\prime}}^{j}\right]_{[u d]} .
$$

3.- Tensor contraction: In the following, we adapt the algorithm introduced in 17 in order to contract the tensors $X^{i j}$ introduced above in a controlled way. The main idea is to express the objects resulting from the contraction of tensors along the first and last column in the 2-D configuration as matrix product states (MPS) and those obtained along the columns $2,3, \ldots, L-1$ as matrix product operators (MPO) 8]. More precisely, we define

$$
\begin{aligned}
\left\langle\mathbb{X}^{1}\right| & :=\sum_{r_{1} \ldots r_{M}=1}^{m} \operatorname{Tr}\left(\mathrm{X}_{r_{1}}^{11} \ldots \mathrm{X}_{r_{M}}^{M 1}\right)\left\langle r_{1} \ldots r_{M}\right| \\
\left|\mathbb{X}^{L}\right\rangle & :=\sum_{l_{1} \ldots l_{M}=1}^{m} \operatorname{Tr}\left(\mathrm{X}_{l_{1}}^{1 L} \ldots \mathrm{X}_{l_{M}}^{M L}\right)\left|l_{1} \ldots l_{M}\right\rangle \\
\mathbb{X}^{j} & :=\sum_{l_{1}, r_{1}, \ldots=1}^{m} \operatorname{Tr}\left(\mathrm{X}_{l_{1} r_{1}}^{1 j} \ldots \mathrm{X}_{l_{M} r_{M}}^{M j}\right)\left|l_{1} \ldots\right\rangle\left\langle r_{1} \ldots\right|,
\end{aligned}
$$

where $m=n$ for $2-\mathrm{D}$ classical systems and $m=\kappa^{2}$ for 1-D quantum systems. These MPS and MPOs are associated to a chain of $M m$-dimensional systems and their virtual dimension amounts to $D=n$. Note that for 2-D classical systems the first and last matrices under the trace in the MPS and MPO reduce to vectors. The partition function (and similarly other correlation functions) reads $Z=\left\langle\mathbb{X}^{1}\left|\mathbb{X}^{2} \cdots \mathbb{X}^{L-1}\right| \mathbb{X}^{L}\right\rangle$. Evaluating this expression iteratively by calculating step by step $\left\langle\mathbb{X}^{j}\right|:=\left\langle\mathbb{X}^{j-1}\right| \mathbb{X}^{j}$ for $j=2, \ldots, L-1$ fails because the virtual dimension of the MPS $\left\langle\mathbb{X}^{j}\right|$ increases exponentially with $j$. A way to circumvent this problem is to replace in each iterative step the MPS $\left\langle\mathbb{X}^{j}\right|$ by a MPS $\left\langle\tilde{\mathbb{X}}^{j}\right|$ with a reduced virtual dimension $\tilde{D}$ that approximates the state $\left\langle\mathbb{X}^{j}\right|$ best in the sense that the norm $\delta K:=\|\left\langle\mathbb{X}^{j}\right|-\left\langle\tilde{\mathbb{X}}^{j}||\right|$ is minimized. Due to the fact that this cost function is multiquadratic in the variables of the MPS, this minimization can be carried out very efficiently [8, 16, 17]; the exponential increase of the virtual dimension can hence be prevented and the iterative evaluation of $Z$ becomes tractable, such that an approximation to the partition function can be obtained from $Z \simeq\left\langle\tilde{\mathbb{X}}^{L-1} \mid \mathbb{X}^{L}\right\rangle$. The accuracy of this approximation depends only on the choice of the reduced dimension $\tilde{D}$ and the approximation becomes exact for $\tilde{D} \geq D^{L}$. As the norm $\delta K$ can be calculated at each step, $\tilde{D}$ can be increased dynamically if the obtained accuracy is not large enough. In the worst case scenario, such as in the NPcomplete Ising spin glasses [18], $\tilde{D}$ will probably have to 
grow exponentially in $L$ for a fixed precision of the partition function. But in less pathological cases it seems that $\tilde{D}$ only has to grow polynomially in $L$; indeed, the success of the methods developed by Nishino [2] in the translational invariant case indicate that even a constant $\tilde{D}$ will produce very reliable results.

4.- Illustration: Bosons in optical lattices: A system of trapped bosonic particles in a 1-D optical lattice of $L$ sites is described by the Bose-Hubbard Hamiltonian [19]

$H=-J \sum_{i=1}^{L-1}\left(a_{i}^{\dagger} a_{i+1}+\right.$ h.c. $)+\frac{U}{2} \sum_{i=1}^{L} \hat{n}_{i}\left(\hat{n}_{i}-1\right)+\sum_{i=1}^{L} V_{i} \hat{n}_{i}$

where $a_{i}^{\dagger}$ and $a_{i}$ are the creation and annihilation operators on site $i$ and $\hat{n}_{i}=a_{i}^{\dagger} a_{i}$ is the number operator. This Hamiltonian describes the interplay between the kinetic energy due to the next-neighbor hopping with amplitude $J$ and the repulsive on-site interaction $U$ of the particles. The last term in the Hamiltonian models the harmonic confinement of magnitude $V_{i}=V_{0}\left(i-i_{0}\right)^{2}$. The variation of the ratio $U / J$ drives a phase-transition between the Mott-insulating and the superfluid phase, characterized by localized and delocalized particles respectively [20]. Experimentally, the variation of $U / J$ can be realized by tuning the depth of the optical lattice [19, 21]. On the other hand, one typically measures directly the momentum distribution by letting the atomic gas expand and then measuring the density distribution. Thus, we will be mainly interested here in the (quasi)-momentum distribution

$$
n_{k}=\frac{1}{L} \sum_{r, s=1}^{L}\left\langle a_{r}^{\dagger} a_{s}\right\rangle e^{i 2 \pi k(r-s) / L} .
$$

Our goal is now to study with our numerical method the finite-temperature properties of this system for different ratios $U / J$. We thereby assume that the system is in a thermal state corresponding to a grand canonical ensemble with chemical potential $\mu$, such that the partition function is obtained as $Z=\operatorname{Tr} e^{-\beta(H-\mu \hat{N})}$. Here, $\hat{N}=\sum_{i=1}^{L} \hat{n}_{i}$ represents the total number of particles. For the numerical study, we assume a maximal particlenumber $q$ per lattice site, such that we can project the Hamiltonian $H$ on the subspace spanned by Fock-states with particle-numbers per site ranging from 0 to $q$. The projected Hamiltonian $\tilde{H}$ then describes a chain of $L$ spins, with each spin acting on a Hilbert-space of dimension $n=q+1$. A Trotter decomposition that turned out to be advantageous for this case is

$$
e^{-\beta(\tilde{H}-\mu \hat{N})}=\left(\hat{V}^{\dagger} \hat{V}\right)^{M}+\mathrm{O}\left[\frac{1}{M^{2}}\right]
$$

with $\tilde{H}=H_{R}+H_{S}+H_{T}, H_{R}=-\frac{J}{2} \sum_{i=1}^{L-1} R^{(i)} R^{(i+1)}$, $H_{S}=-\frac{J}{2} \sum_{i=1}^{L-1} S^{(i)} S^{(i+1)}, H_{T}=\sum_{i=1}^{L} T^{(i)}, R^{(i)}=$ $\tilde{a}_{i}^{\dagger}+\tilde{a}_{i}, S^{(i)}=-i\left(\tilde{a}_{i}^{\dagger}-\tilde{a}_{i}\right), T^{(i)}=\frac{1}{2} \tilde{n}_{i}\left(\tilde{n}_{i}-1\right)+V_{i} \tilde{n}_{i}$

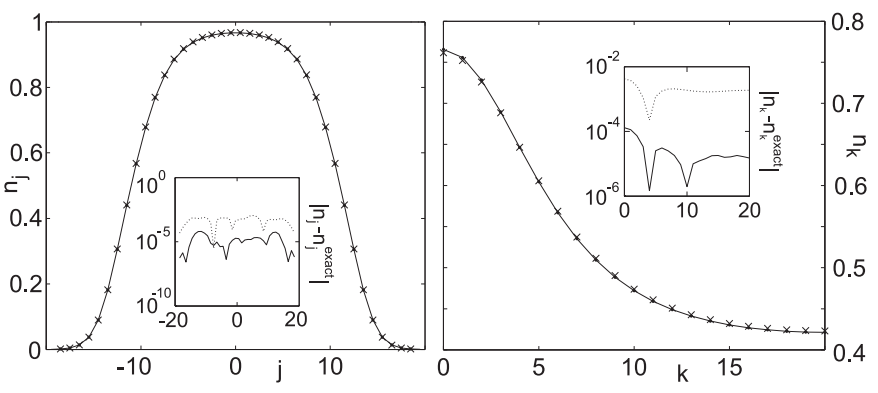

FIG. 1: Density and (quasi)-momentum distribution in the Tonks-Girardeau gas limit, plotted for $\beta J=1, L=40$, $N=21$ and $V_{0} / J=0.034$. The dots (crosses) represent the numerical results for $\tilde{D}=2(\tilde{D}=8)$ and the solid line illustrates the exact results. From the insets, the error of the numerical results can be gathered.

and $\hat{V}=e^{-\frac{\beta}{2 M} H_{R}} e^{-\frac{\beta}{2 M} H_{S}} e^{-\frac{\beta}{2 M}\left(H_{T}-\mu \hat{N}\right)} . \quad \tilde{a}_{i}^{\dagger}, \tilde{a}_{i}$ and $\tilde{n}_{i}$ thereby denote the projections of the creation, the annihilation and the number operators $a_{i}^{\dagger}, a_{i}$ and $n_{i}$ on the $q$-particle subspace. The decomposition (1) of all two-particle operators in expression (2) then straightforwardly leads to a set of 4 -index tensors $X_{l r u d}^{i j}$, with indices $l$ and $r$ ranging from 1 to $(q+1)^{3}$ and indices $u$ and $d$ ranging from 1 to $q+1$. Note that the typical second order Trotter decomposition with $H=H_{\text {even }}+H_{\text {odd }}$ would make the indices $l$ and $r$ range from 1 to $(q+1)^{6}$.

Let us start out by considering the limit $U / J \rightarrow \infty$ in which double occupation of single lattice sites is prevented and the particles in the lattice form a TonksGirardeau gas [13]. In this limit, the Bose-Hubbard Hamiltonian maps to the Hamiltonian of the exactly solvable (inhomogeneous) XX-model, which allows to benchmark our algorithm. The comparison of our numerical results to the exact results can be gathered from fig. 1] Here, the density and the (quasi)-momentum distribution are considered for the special case $\beta J=1, L=40$, $N=21$ and $V_{0} / J=0.034$. The numerical results shown have been obtained for Trotter-number $M=10$ and two different reduced virtual dimensions $\tilde{D}=2$ and $\tilde{D}=8$. The norm $\delta K$ was of order $10^{-4}$ for $\tilde{D}=2$ and $10^{-6}$ for $\tilde{D}=8[26]$. From the insets, it can be gathered that the error of the numerical calculations is already very small for $\tilde{D}=2$ (of order $10^{-3}$ ) and decreases significantly for $\tilde{D}=8$. This error can be decreased further by increasing the Trotter-number $M$.

As the ratio $U / J$ becomes finite, the system becomes physically more interesting, but lacks an exact mathematical solution. In order to judge the reliability of our numerical solutions in this case, we check the convergence with respect to the free parameters of our algorithm $(q$, $\tilde{D}$ and $M)$. As an illustration, the convergence with respect to the parameter $q$ is shown in figure 2 In this figure, the density and the (quasi)-momentum distribution are plotted for $q=2,3$ and 4 . We thereby assume 


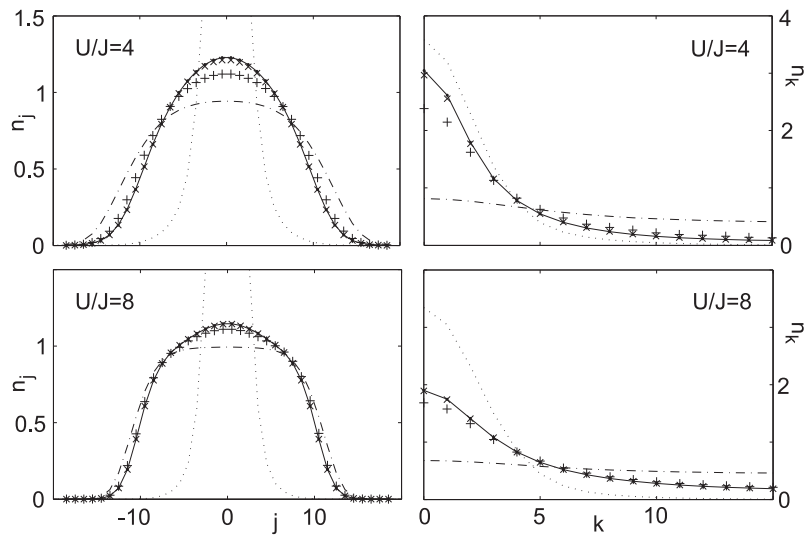

FIG. 2: Density and (quasi)-momentum distributions for interaction strengths $U / J=4$ and 8 . Here, $\beta J=1, L=40$, $N=21$ and $M=10$. Numerical results were obtained for $q=2$ (plus-signs), $q=3$ (crosses) and $q=4$ (solid line). For comparison, the distributions for $U / J=0$ (dotted lines) and $U / J \rightarrow \infty$ (dash-dotted lines) are also included.

that $\beta J=1, L=40$ and $N=21$ and consider interaction strengths $U / J=4$ and 8 . The harmonic potential $V_{0}$ is chosen in a way to describe Rb-atoms in a harmonic trap of frequency $\mathrm{Hz}$ (along the lines of [13]). We note that we have taken into account that changes of the ratio $U / J$ are obtained from changes in both the on-site interaction $U$ and the hopping amplitude $J$ due to variations of the depth of the optical lattice. The numerical calculations have been performed with $M=10$ and $\tilde{D}=q+1$. From the figure it can be gathered that convergence with respect to $q$ is achieved for $q \geq 3$.

We now use our numerical algorithm to study a physical property of interacting bosons in an optical lattice, namely the full width at half maximum (FWHM) of the (quasi)-momentum distribution. It has been predicted that the FWHM shows a kink at zero temperature 22, 23, 24]. This kink is an indication for a Mottsuperfluid transition, since the FWHM is directly related to the inverse correlation length. Experiments have also revealed this kink 12, 14]; they are, however, performed at finite temperature, something we can study with our algorithm. In figure 3 we plot the numerical results for the FWHM as a function of $U / J$ for three different (inverse) temperatures $\beta J=0.5,1$ and 2 . The physical parameters $L, N$ and $V_{0}$ are thereby chosen as in the previous case. The numerical results have been obtained for $M=10, q=4$ and $\tilde{D}=q+1$. For each temperature, three different regions can be distinguished: the superfluid region with constant FWHM, the Mott-region with linearly increasing FWHM and an intermediate region in which both phases coexist. The value $U / J$ at which the Mott-region starts increases with increasing temperature, which is consistent with the criteria $U \gg k_{B} T, J$ for the appearance of the Mott-phase. This behaviour could be
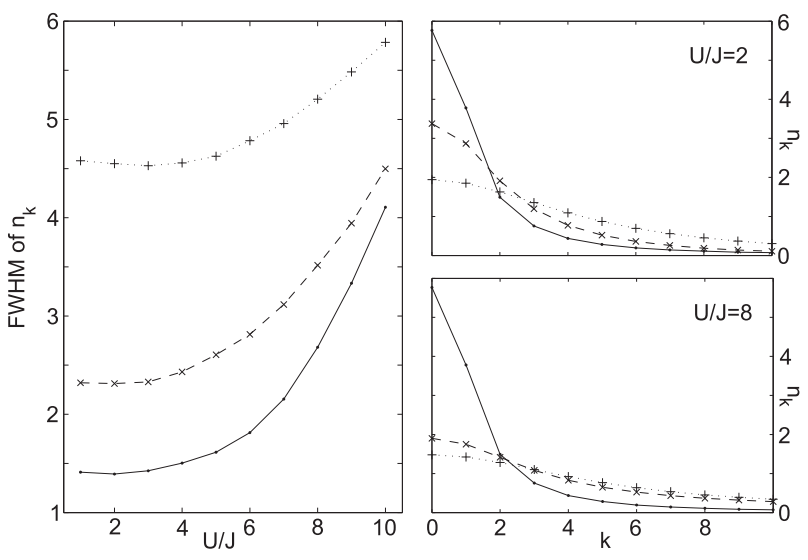

FIG. 3: FWHM of the (quasi)-momentum distribution as a function of $U / J$, calculated for temperatures $\beta J=0.5$ (plussign), $\beta J=1$ (crosses) and $\beta J=2$ (dots). The corresponding (quasi)-momentum distributions for $U / J=2$ and $U / J=8$ are illustrated in the plots at the right-hand side.

easily observed in present experiments.

In summary, we have presented a numerical method for the investigation of thermal states of inhomogeneous 2-D classical and 1-D quantum systems. We have illustrated the usefulness of this method by applying it to a system of trapped bosonic particles in a 1-D optical lattice - which is of current experimental interest. For this system, we have studied the error and the convergence of the method. In addition, we have used the method to study some physical properties of this system and we have obtained results which can be verified experimentally.

We thank M. A. Martin-Delgado and D. Porras for discussions. Work supported by the DFG, european projects (IST and RTN) and the Kompetenznetzwerk der Bayrischen Staatsregierung Quanteninformation.

[1] M. Troyer and U. Wiese (2004), cond-mat/0408370.

[2] T. Nishino, J. Phys. Soc. Jpn. 64, 3598 (1995).

[3] S. R. White, Phys. Rev. Lett 69, 2863 (1992).

[4] U. Schollwöck (2004), cond-mat/0409292.

[5] R. J. Bursill, T. Xiang, and G. A. Gehring, J. Phys. Conden. Matter 8, L583 (1996).

[6] N. Shibata, J. Phys. Soc. Jpn. 66, 2221 (1997).

[7] X. Wang and T. Xiang, Phys. Rev. B 56, 5061 (1997).

[8] F. Verstraete, J. J. García-Ripoll, and J. I. Cirac, Phys. Rev. Lett 93, 207204 (2004), cond-mat/0406426.

[9] M. Zwolak and G. Vidal, Phys. Rev. Lett 93, 207205 (2004), cond-mat/0406440.

[10] M. Fannes, B. Nachtergaele, and R. F. Werner, Comm. Math. Phys. 144, 443 (1992).

[11] F. Verstraete, M. A. Martin-Delgado, and J. I. Cirac, Phys. Rev. Lett 92, 087201 (2004), quant-ph/0311087.

[12] T. Stöferle, H. Moritz, C. Schori, M. Köhl, and 
T. Esslinger, Phys. Rev. Lett. 92, 130403 (2004), condmat/9312440.

[13] B. Paredes, A. Widera, V. Murg, O. Mandel, S. Fölling, I. Cirac, G. V. Shlyapnikov, T. W. Hänsch, and I. Bloch, Nature 429, 277 (2004).

[14] M. Köhl, T. Stöferle, H. Moritz, C. Schori, and T. Esslinger, Appl. Phys. B 79, 1009 (2004), condmat/0411473.

[15] M. Girardeau, J. Math. Phys. 1, 516 (1960).

[16] F. Verstraete and J. I. Cirac (2004), cond-mat/0407066.

[17] F. Verstraete, D. Porras, and J. I. Cirac, Phys. Rev. Lett 93, 227205 (2004), cond-mat/0404706.

[18] F. Barahona, J. Phys. A 15, 3241 (1982).

[19] D. Jaksch, C. Bruder, J. I. Cirac, C. W. Gardiner, and P. Zoller, Phys. Rev. Lett 81, 3108 (1998), condmat/9805329.
[20] M. P. Fisher, P. B. Weichmann, G. Grinstein, and D. S. Fisher, Phys. Rev. B 40, 546 (1989).

[21] H. P. Büchler, G. Blatter, and W. Zwerger, Phys. Rev. Lett. 90, 130401 (2003).

[22] C. Kollath, U. Schollwöck, J. von Delft, and W. Zwerger, Phys. Rev. A 69, 031601 (2004), cond-mat/0310388.

[23] S. Wessel, F. Alet, S. Trebst, D. Leumann, M. Troyer, and G. G. Matrouni (2004), cond-mat/0411473.

[24] L. Pollet, S. M. A. Rombouts, and P. J. H. Denteneer, Phys. Rev. Lett. 93, 210401 (2004), cond-mat/0408596.

[25] Note that in practice, it will be desirable to use the higher order versions of the Trotter decomposition.

[26] We note that we have stopped our iterative algorithm at the point the variation of $\delta K$ was less than $10^{-8}$. 